\title{
Time marches on...but not for sea urchins
}

Aging is inevitable. With time, cells lose their ability to regenerate and tissues and organs succumb to wear and tear. Behind the stiff joints and wrinkles are changes accumulating in genes involved with DNA replication and transcription that disrupt cell proliferation and eventually lead to senescence, wherein cells stop actively dividing, or apoptosis, programmed cell death. Current theory links aging to an organism's lifespan and life history. Shortlived species that tend to reproduce early and rapidly are thought to not invest the resources and energy into cellular maintenance and repair and thus age quickly, while the aging process may be more gradual in species with longer lifespans and later reproductive maturity. Andrea Bodnar and James Coffman set out to document contrasting aging phenomena in sea urchins with vastly different lifespans in a recent study published in Aging Cell (15, 778-787; 2016).

Lifespans in sea urchins can vary greatly by species, with some surviving only a few short years while others are capable of living for decades with negligible senescencegrowing, reproducing, and regenerating to the very end. Bodnar and Coffman were interested in three species: the short-lived Lytechinus variegatus, the middle-aged Strongylocentrotus purpuratus, and the centenarian Mesocentrotus franciscanus. Dividing each of their subject species into "young" and "old" groups, they tested the expression of genes involved in cell proliferation, telomere maintenance, and multipotency in several different tissue types. They also studied the urchins' ability to regrow their spines and tube feet. By any measure, the youth showed no major advantages over their elders, regardless of species. As Coffman explained in a press release, “... aging is not inevitable: sea urchins don't appear to age, even when they are shortlived. Because these findings were unexpected in light of the prevailing theories about the evolution of aging, we may have to rethink theories on why aging occurs."

Although one might be hard-pressed to find similarities between an urchin

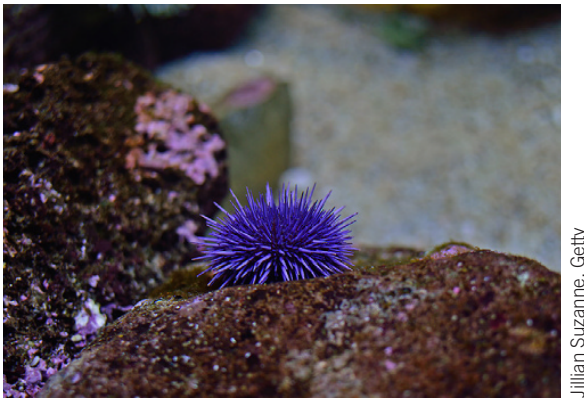

and a human, they actually share a closer phylogenetic relationship than humans and other kinds of invertebrates. The genes analyzed in this study are either conserved in humans or similar in function. Urchin embryos have long been used to study life at the earliest developmental stages but adults are now becoming models for understanding life's progression to the end. Understanding how sea urchins are able to avoid the ravages of time may inform the evolutionary theory of aging as well as the underlying process in humans and other vertebrates.

Ellen P. Neff

\section{PREDICTING DISEASE GENOTYPES FROM BEHAVIOR}

Genome-wide association studies, which quantify the genetic variation between individuals with and without a specific trait-or phenotype-provide an important avenue for discovering multiple genes associated with a single disease. An alternative approach, designed to link multiple phenotypes to a single genetic variant, is the phenome-wide association study. Although previous research using patient medical records has demonstrated proof-of-principle for this latter approach, the narrow scope of these studies does not reflect the complex phenotypic effects that variation in a single gene can produce.

With the rapid expansion in technology for recording longitudinal health and behavioral data, there is growing interest in predicting an individual's genome from behavioral traits alone, but methods for analyzing these complex data sets are still limited. Computational methods, like machine learning algorithms, offer the possibility of breaking down high-dimensional data into distinct features that can enable accurate predictions, and groups of scientists are now applying these approaches to see just how well they can predict a genotype from phenotypic data alone.

Using a mouse model of Huntington's disease, which has well known but complex abnormalities in cognitive and motor functions owing to CAG-repeats in the huntingtin HTT gene, Vadim Alexandrov at PsychoGenics Inc. and colleagues applied computer vision and support vector machines to behavioral data and were able to accurately predict mutant from wild-type mice (Nat. Biotechnol. 34, 838-844; 2016). But, the team of researchers went a step further. Because the length of CAG-repeats affects the severity and timing of onset of Huntington's symptoms, the team evaluated their method to see if they could distinguish between mutant mice with different CAG-repeat lengths. Using three groups of mice with defined CAG repeats, and 'blinded mice' with unknown CAG-repeat lengths, the team found they could accurately distinguish between the defined groups with $100 \%$ accuracy and were also able to predict the length of CAGrepeats in the blinded mouse group.

The remarkable accuracy of their analysis raises the hope that behavioral traits might provide sufficient information to predict an individual's genotype. The sensitivity of their method could also allow researchers to use more subtle mouse models of Huntington's, which better mimic human clinical cases, when testing candidate drug treatments, increasing the translational impact of genetically modified mouse models. Whether used with animal models of disease, or directly applied to human data, the adoption of computational methods for analyzing complex phenotypic data will likely increase, especially as new technologies continue to make behavioral data easier to capture.

Dustin M. Graham 